Meta

Journal des traducteurs

Translators' Journal

\title{
Humour et traduction au contact des cultures
}

\section{Anne-Marie Laurian}

Volume 34, numéro 1, mars 1989

Humour et traduction

Humour and Translation

URI : https://id.erudit.org/iderudit/003418ar

DOI : https://doi.org/10.7202/003418ar

Aller au sommaire du numéro

Éditeur(s)

Les Presses de l'Université de Montréal

ISSN

0026-0452 (imprimé)

1492-1421 (numérique)

Découvrir la revue

Citer cet article

Laurian, A.-M. (1989). Humour et traduction au contact des cultures. Meta,

34(1), 5-14. https://doi.org/10.7202/003418ar d'utilisation que vous pouvez consulter en ligne.

https://apropos.erudit.org/fr/usagers/politique-dutilisation/ 


\section{HUMOUR ET TRADUCTION AU CONTACT DES CULTURES*}

ANNE-MARIE LAURIAN

C.N.R.S., Paris, France

L'humour est souvent considéré comme une sorte de processus magique réservé aux initiés, un pouvoir de création ou une capacité de compréhension demandant un long apprentissage pour être mis en œuvre. On admire l'humour des autres, on essaye d'en faire preuve, et on se heurte à l'indifférence ou à l'incompréhension, voire au malentendu. Si ce phénomène se produit au sein d'une pratique langagière monolingue, à plus forte raison risque-t-il de se faire sentir lorsque les locuteurs sont confrontés à des langues étrangères ou lorsqu'ils tentent de s'exprimer en une langue seconde. Si cette magie de l'humour a son origine dans les performances linguistiques, la traduction de l'humour de langue à langue peut sembler compromise. Pourtant elle est possible.

En examinant différentes composantes linguistiques et culturelles d' $d^{2}$ histoires drôles», on va tenter de proposer un classement des difficultés de la traduction de l'humour. Sur cette base on pourra suggérer quelques orientations pour un enseignement des langues vivantes donnant une place accrue à l'humour.

Une première difficulté apparaît dans l'attribution d'une signification précise au terme même «humour». Le Petit Robert indique: «Forme d'esprit qui consiste à présenter la réalité de manière à en dégager les aspects plaisants et insolites» (édition 1967). Mais Robert Escarpit ${ }^{1}$ donne à l'introduction de son ouvrage le titre «L'Impossible définition», et, en sous-titre : «I - Pourquoi nous ne pouvons définir l'humour». Indiquant les deux orientations sémantiques du mot français qui ont donné lieu d'ailleurs historiquement à une différenciation phonétique entre «humeur» et «humour», il nous conduit tout naturellement à prendre en considération la forme anglaise du mot et les deux «non-définitions» données par l'Encyclopæedia Britannica dans sa première édition de 1771:

Humour : see Fluid

Humour: see Wit

On trouve ces deux axes dans un dictionnaire anglo-américain actuel: sous l'entrée Humor, on a: «(...) 2. a) A person's disposition or temperament. b) A mood; state of mind. (...) 4. The quality that makes something seem funny, amusing or ludicrous; comicality. 5. a) The ability to perceive, appreciate or express what is funny, amusing or ludicrous. (...)» (Webster's New World Dictionary, College Edition, 1959.)

Les récits très brefs que l'on trouve dans de nombreux journaux américains sous la plume d'humoristes professionnels ou non, les cours de "creative writing" ou "creative humor writing» qui sont dispensés dans certaines universités américaines, ou les billets que l'on trouve dans quelques rares journaux français participent du premier emploi de humour/humor (graphie britannique ou graphie américaine): l'auteur exprime souvent un mouvement d'humeur - mais il l'exprime avec humour, ce qui nous renvoie sur le second emploi du mot. Sous le titre «Humour» on trouve souvent dans la presse française des dessins ou des caricatures sous-titrés ou non, et dans les rayons des librairies c'est sous ce titre que l'on trouve les recueils de bandes dessinées pour enfants ou pour adultes, 
ainsi que les recueils de blagues ou de «bons mots», compilations de blagues populaires ou créations d'auteurs spécialisés. On voit donc que dans l'usage quotidien, le mot «humour» s'applique à nombre de productions diverses. Ici nous nous intéresserons particulièrement aux jeux de mots, blagues, histoires drôles qui apparaissent dans des recueils spécialisés.

L'humour est souvent considéré comme intraduisible, et pourtant on le traduit. Parfois il est très aisé à traduire, parfois très difficile. C'est l'effort, et bien souvent l'imagination et la créativité nécessaires à sa traduction qui lui donnent cette image d' «objet intraduisible». Reconnaissons cependant qu'il existe des cas où la difficulté semble effectivement insurmontable - «semble» seulement car nous sommes persuadée qu'il pourra se trouver un jour un traducteur plus habile, mieux entraîné, ou plus inventif qui traduira ce qui était considéré comme désespéré auparavant. Il en est de la traduction comme des sports : la limite semble pouvoir toujours être reculée.

Ces variations de jugements portés sur la traductibilité de l'humour proviennent, à notre sens, du caractère ressenti immédiatement comme doublement constitué de l'humour: caractère linguistique et caractère culturel. La combinaison dans des proportions variables de ces deux facteurs rend la traduction plus ou moins aisée, comme cela apparaitra dans les exemples ci-dessous.

Les théories classiques de la traduction nous apprennent ce qu'une traduction devrait faire et comment elle devrait le faire, à savoir qu'un texte écrit en une langue L2 devrait exprimer le même sens qu'un texte écrit en une langue d'origine L1; et pour exprimer le même sens, il convient de passer des mots aux idées, de la syntaxe aux concepts, des formulations aux sémantismes, et puis de refaire le chemin en direction inverse vers la langue-cible. Bien évidemment nous avons ici légèrement schématisé... Il n'empêche que la notion de «sens» est communément utilisée par les théoriciens ou les descripteurs de la traduction. En ce qui concerne les «pièces d'humour», qu'il s'agisse de blagues, plaisanteries de conversation, récits ou images sous-titrées, où est le sens? Quelle est la signification de «sens» lorsqu'il s'agit d'humour? Y a-t-il des jeux «gratuits» avec les mots? Une explication des mots et des référents ne couvre pas l'entière signification. Les intentions de l'auteur et les données du texte se mêlent en général à un ensemble de connotations ou de références incluant l'histoire, la politique, les attitudes, les modes de vie, les traditions, la littérature, la science, et toutes sortes de domaines dans lesquels les locuteurs natifs sont immergés en permanence et qui varient de contrée à contrée, donc de langue à langue dans la mesure où la géographie et la linguistique sont solidaires.

L'humour est souvent fondé sur une connivence entre l'auteur et le lecteur, le locuteur et l'auditeur. Ce sont les éléments de cette connivence, de ces connaissances communes et de ces intentions partagées que nous allons tenter de décrire à travers quelques exemples particuliers.

Commençons par quelques blagues simples, quelques «elephant jokes». Celles que nous présentons ici sont extraites d'un recueil établi par Des MacHale?

\section{1. - What has fifty legs but cannot walk? \\ - Half a centipede.}

Cette devinette est très facile à comprendre et à traduire. Il suffit de modifier l'indication numérique : «fifty» se «traduira» en «cinq cents» de façon à obtenir un «millepattes» pour l'anglais «centipede». («Qu'est-ce qui a cinq cents pattes et qui ne peut pas marcher? - La moitié d'un mille-pattes.») Le chiffre indiqué ne correspondant à aucune réalité extralinguistique, la blague repose sur un savoir linguistique partagé par le conteur et les auditeurs. 


\section{2. - Why is an elephant like a pink sausage? \\ - Neither of them can climb a tree.}

Il n'y a aucun problème d'ordre linguistique pour traduire cette blague : ni la forme interrogative ni la forme comparative, ni le vocabulaire utilisé ne se heurtent à, par exemple, une absence de correspondant en français. («Pourquoi l'éléphant ressemble-t-il à une saucisse rose? - Aucun des deux ne peut grimper à un arbre.») Cependant cette devinette d'enfant ne peut être comprise que de ceux qui savent à quoi ressemble un éléphant et à quoi ressemble une saucisse. Amuserait-elle autant un enfant esquimau qui n'a jamais vu d'éléphant et qui n'a pas l'habitude de manger des saucisses qu'un enfant européen ou américain qui fréquente les zoos en savourant des hot-dogs au ketchup ou à la moutarde? En traduisant pour un enfant d'une autre culture, peut-être conviendrait-il de changer l'éléphant en baleine, l'arbre en iceberg, la saucisse en «steak» de phoque. Quant à la couleur de la saucisse, il faudrait tenir compte des associations («pink panther» par exemple) pour en décider tout en sachant que si l'on adoptait le français «saucisse de Francfort, Strasbourg ou Toulouse», on aurait du mal à créer un lien entre un nom de ville et un nom d'animal grimpant à un arbre.

\section{3. - What is grey, has four legs, a trunk and flies? - A dead elephant.}

Ici, l'un des éléments lexicaux possède deux référents, et c'est la perception par l'auditeur de ce «double-sens» qui crée la réaction d'amusement. En effet, «flies» renvoie au verbe «to fly» («voler») et au substantif «fly» («mouche»). Si l'on ne peut trouver en français d'équivalent combinant ces deux sémantismes, il conviendra de créer un effet similaire en s'appuyant sur le double sémantisme d'un lexème appartenant à un champ lexical peut-être totalement différent. Le lexème «vol-» en français offre une double orientation de type similaire : on peut «voler» dans les airs et «voler» un objet qui appartient à quelqu'un d'autre. Sur cette base, en modifiant l'éléphant mort en éléphant cambrioleur on pourrait obtenir la devinette: «Qu'est-ce qui est gris, qui a quatre pattes, une trompe et qui vole? - Un éléphant cambrioleur». L'effet de surprise proviendrait d'une préparation similaire à l'anglais (avec «flies / vole» qui spontanément dans ce contexte est compris comme le mouvement aérien) et d'un choc au moment de la réponse qui renvoie vers une autre aire sémantique. On perd l'aspect visuel et concret des mouches sur l'éléphant mort mais on conserve le type de jeu lexical.

$$
\begin{aligned}
& \text { 4. - How would you get rid of a white elephant? } \\
& \text { - Give it to a jumbo sale. }
\end{aligned}
$$

La difficulté de traduction de cette devinette repose sur une attente particulière suscitée par l'indication de la couleur de l'éléphant; elle se résoud en un jeu qui ne tient pas compte de cette couleur mais donne une sorte d'équivalent lexical ("elephant / jumbo»); cependant cette équivalence est un américanisme, et l'expression «jumbo sale» est britannique (correspondant à «garage sale» ou «yard sale» aux États-Unis). On se trouve donc confronté, pour la traduction, à une double difficulté : le rendu de la surprise fondée sur une sorte de simplicité extrême (et qui permettrait par exemple : «Comment peut-on se débarrasser d'un éléphant blanc? - En le vendant au marché des éléphants») et le rendu culturel de cette réalité vivante que sont les ventes d'objets domestiques devenus inutiles le dimanche devant la maison, réalité exprimée différemment de part et d'autre de 1'Atlantique (ce qui implique une prise en compte du destinataire). On peut suggérer le «marché aux puces» pour jouer sur les dimensions, mais il faudrait une relation motivée entre «elephant» et «puces» pour que l'histoire produise un sourire. Sur l'idée de la 
vente-débarras, on peut suggérer, à partir de l'expression «mettez-le en solde», la formulation: «Affichez: Soldes monstres», ce qui aurait pour avantage de créer une relation «éléphant / monstre». On avait aussi pensé à utiliser le nom d'une chaîne française de supermarchés ou d'hypermarchés: Mammouth, mais ceci introduirait une réalité trop localisée géographiquement, incompréhensible au francophone d'autres régions (mais n'existe-t-il pas entre les différentes régions francophones le même genre de différences qu'entre les différentes régions anglophones?)**

\section{What is grey and sings?} Harry Elephonte.

Pour comprendre cette devinette, il ne suffit pas de savoir la langue, il faut aussi connaître l'histoire de la chanson, et il est fort vraisemblable que les moins de vingt ans, sauf à posséder une certaine culture artistique, n'ont jamais entendu parler de Harry Belafonte. Pour traduire cette blague, deux problèmes se posent donc: rendre le jeu de sonorités «elephant / Belafonte», et s'adresser à un public français actuel. C'est une double traduction: dans l'espace et dans le temps, que l'on devra créer. Une traductiontransposition a été proposée par une personne qui a réussi à combiner le nom d'une chanteuse française célèbre et le nom d'un animal de couleur grise (on s'éloigne des éléphants mais on reste parmi les animaux): "Qu'est-ce qui est gris et qui chante? - Nana Masouris» ${ }^{3}$.

Ce type de plaisanterie reposant sur des références culturelles propres à une contrée ou à une période mène, dans une hiérarchie allant du moins marqué culturellement au plus marqué culturellement, à des blagues qui demandent de plus en plus d'imagination au traducteur. Nous en donnons ici quelques-unes tirées d'un recueil par Herb True 4 .

6. The doctor gave his patient six months to live. The patient couldn't pay his bill so the doctor gave him another 6 months.

Le délai-crédit octroyé par le médecin-banquier est facile à percevoir, mais l'histoire prend encore plus de relief dans le contexte américain de la «vie à crédit» (cartes de crédit et médecins effectivement payés avec certains délais, ce qui est inconcevable en France, sauf dans des situations très particulières).

\section{A pessimist is one who can only see the hole in the doughnut.}

Il n'y a pas ici de problème lexical ou syntaxique, mais la réalité référée par «doughnut» n'existe pas en France: les beignets ni les «krapfen» (vendus sur les boulevards) n'ont de trou central. La forme de tore est une spécialité américaine. Comment rendre donc la vision du pessimiste en français ? On songe à la classique bouteille à moitié vide opposée à la bouteille à moitié pleine, mais cette image est beaucoup trop connue pour créer le moindre effet d'amusement. On a cherché une spécialité culinaire typiquement européenne et présentant un trou ou des trous de façon à conserver l'image donnée par le «doughnut» à trou central. L'image du gruyère a été proposée, ce qui donnerait: «Le pessimiste est celui qui ne voit dans le gruyère que les trous»5.

8. The world is so full of problems that, if Moses came down from Mount Sinai today, two of the tablets he would be carrying would be aspirin.

On se trouve ici face à des lexies figées: les «tables de la loi» ne peuvent être que des «tables» et non des «tablettes», et les aspirines sont des «cachets» et non des «tablettes» (même si certains médicaments existent sous la forme de tablettes). Pour 
conserver un jeu sur «cachet» on peut imaginer de transformer Mö̈se en comédien, mais il faudra aussi supprimer le mont Sinaï, et l'ensemble du choc entre le poids historique et religieux de Moïse descendant du mont Sinaï et la légèreté des aspirines serait perdu. $\grave{A}$ ce jour nous n'avons pas trouvé de solution acceptable.6.

Dans un autre cadre, la culture commune au locuteur et à l'auditeur n'est plus du domaine des données historiques, culinaires, linguistiques, etc. (données encyclopédiques ou données expérimentales), mais du domaine du sentiment stylistique. Ainsi par exemple les Lois de Murphy ne sont amusantes que pour celui qui, au-delà de l'énoncé d'une vérité générale (ce qu'est une loi) sent le style de l'énonciation scientifique appliqué à des phénomènes prosaïquement quotidiens. En voici quelques exemples ${ }^{7}$ :

9. Law of the perversity of nature : You cannot successfully determine beforehand which side of the bread to butter.

10. Jenkinson's law: It won't work.

11. Sattinger's law: It works better if you plug it in.

12. Fett's law of the lab: Never replicate a successful experiment.

13. Lubarsky's law of cybernetic entomology: There's always one more bug.

Dans tous ces énoncés, l'humour naît de la généralisation et du style de l'énonciation. Les mêmes énoncés prononcés dans des situations particulières ne seraient pas drôles; mais présentés comme des lois valables dans toutes les situations, ils prennent un aspect scientifique qui entre en «pseudo-contradiction» avec le contenu. La tartine beurrée en est l'exemple maximal: chacun a pu faire l'expérience de faire tomber par terre une tartine beurrée ou «confiturée»; s'il dit «pas d'chance!» lorsque le côté recouvert touche le sol, il n'y a rien de drôle; s'il fait de son accident un modèle expérimental d'une loi naturelle, alors l'humour naît. Les données linguistiques sont simples à traduire; le style est l'essentiel. Encore faut-il que la situation stylistique équivalente existe dans la langue-cible. C'est le cas du français («Loi de la perversité de la nature: il est impossible de déterminer à l'avance quel côté du pain il faut beurrer»). Mais on peut imaginer une civilisation qui non seulement ne connaîtrait pas la tartine mais qui, de plus, ne posséderait pas une science expérimentale parvenue au stade d'observation des lois naturelles régissant le comportement des tartines. Dans ce cas l'énoncé d'une telle loi, quelle que soit la langue en laquelle il serait fait, n'aurait aucune valeur.

La loi donnée sous le $n^{\circ} 10$ semble résumer les autres. C'est la constatation de bon sens de tout scientifique sceptique arrivé en fin de carrière et osant les généralisations au futur. Chacun de nous a connu quelques-uns de ces pessimistes. Mais (heureusement) la science avance malgré eux (ou grâce à eux - selon le principe du défi - -). Elle est belle par sa généralité même qui semble totalement insurpassable. Aucun problème de traduction apparemment ( «Ça ne marchera pas»), et pourtant selon le style scientifique utilisé pour chaque langue, il faudra «aménager» l'énoncé (par exemple, pour le français : «Ça ne peut pas marcher», «Il a été prouvé que ça ne peut fonctionner», «Cela ne fonctionnera pas»).

La loi $n^{\circ} 11$ est également une simple constatation de bon sens si l'on imagine les situations concrètes où cet énoncé pourrait être dit: lampe, radio, télévision, appareil ménager, ctc., qui ne fonctionne pas et contre lequel l'utilisateur potentiel commence à 
grogner, voire à s'énerver, avec une personne présente intervenant pour lui donner ce conseil: «plug it in». Une formulation atténuée du conseil pourrait prendre la forme de l'énoncé donné: «it works better if you plug it in»; on l'imagine prononcé avec un sourire aux lèvres et une légère ironie. L'humour provient de la transformation du simple conseil en loi générale. Et de la constatation qu'effectivement dans la plupart des situations qui pourront nous venir à l'esprit, la loi sera vérifiée.

L'énoncé $\mathrm{n}^{\circ} 12$ fait référence à une réalité de la vie des laboratoires de recherche et semble faire allusion à une impossibilité d'être réellement scientifique. Il y a une contradiction entre ce qu'on attend théoriquement de la science (l'infinie reproductibilité). Il y a également un jeu sur le sens de «loi»: observation générale (tournée vers le passé) ou ligne de conduite à suivre (pour le futur). Ces deux orientations sémantiques se trouvent dans l'usage courant de «loi» d'une part en sciences exactes ou expérimentales et d'autre part en droit. De plus on peut y voir une règle psychologiquement utile et raisonnable. Toutes ces connotations font que, même si la traduction de l'anglais au français ne semble pas poser de problème linguistique, on pourra rencontrer des difficultés au passage vers d'autres langues qui, par exemple, dissocieraient l'expression lexicale du concept de norme (vérité statistique ou comportement à adopter).

Enfin la loi donnée sous le nom de Lubarsky comme «loi de l'entomologie cybernétique» se présente par son titre même comme humoristique. Elle présente une difficulté de traduction en français, surtout si l'on tient compte des recommandations, voire de la normalisation, indiquées par les commissions ministérielles de terminologie françaises. Chacun sait qu'un "bug" est le terme utilisé par les informaticiens pour désigner un ennui, une difficulté, une gêne, une panne, etc. dans les programmes ou dans le fonctionnement de l'ordinateur. Parfois on sait aussi que ce mot désigne en anglais un insecte tel que, par exemple, le scarabée, le hanneton, la punaise, etc. Mais si l'on peut s'exclamer «oh, punaise !» lorsqu'on a un ennui, ce n'est pas en référence à l'insecte. Si l'on traduit «exactement», on dira: «il y a toujours un beugue de plus», «il y a toujours encore un beugue». Ce terme a été adopté par les commissions compétentes de terminologie française. Mais alors le titre en référence à l'entomologie n'a plus aucun sens. Nous suggérons de changer de domaine scientifique et de passer en agriculture par exemple: «Loi de l'arboriculture cybernétique: Il y a toujours un pépin supplémentaire».

On remarque au passage de l'expression des lois de l'anglais vers le français un changement de la formulation verbale: l'adresse à l'impératif et la forme de $2^{\mathrm{e}}$ personne ont intérêt à être modifiées en formulation de $3^{\mathrm{e}}$ personne dite impersonnelle par «il» ou par «on»8. Les discours scientifiques français privilégient les formes dépersonnalisées. En conséquence, pour en respecter le style, le traducteur devra se conformer aux normes de l'usage français'.

L'exemple suivant, tiré d'un recueil de Kenneth Edwards 10 met également en jeu le style scientifique, ceci en liaison avec une double orientation sémantique de «to live on». Si l'ambiguïté peut être rendue en français, cependant l'ambiguïté risque de n'être pas respectée.

14. A scientist told an audience during his lecture: «150 000 germs can live on a pound note for years». «Good heavens, said one listener, they' re cleverer than I am, I can hardly do it for an hour».

Si l'on pense à la traduction «vivre sur», on pourra rendre l'ambiguité, mais la formulation la plus fréquente pour un budget personnel serait «vivre de». De plus, la forme active du verbe gêne le français qui, dans la situation d'un exposé scientifique, aurait spontanément utilisé une forme dite inverse ou impersonnelle («peuvent se maintenir 
sur» ou «on observe la présence de» ou une autre forme de ce type). Ici encore un phénomène d'usage stylistique interfêre avec la traduction immédiate.

Lorsque l'humour naît d'un double jeu sur la langue et sur la réalité à laquelle se réfèrent les mots, alors la traduction présente des difficultés parfois difficiles à surmonter. En effet si le présupposé est une connaissance de faits de civilisation, il faut que ces connaissances soient acquises au préalable. Peut-on raconter une histoire drôle en y adjoignant des explications? Une introduction éclairante? Des notes de bas de page? Cela serait tout simplement de l'assassinat de blague («witzemörder») dans la majorité des cas. Voici quelques exemples pour illustrer cette idée. Ils sont tirés d'un recueil de Clement de Wrobleskyll.

15. Eine japanische Delegation besucht die DDR. Am Schluß der Besuchreise wird der Leiter der Delegation gefragt, was die Delegation besonders beeindruckt hat.

«Am meisten», sagt der Leiter der Delegation, "haben uns in der DDR die wunderschönen Museen beeindruckt : Pergamon, Robotron, Pentakon...»

(Traduction proposée :

À la fin d'une visite effectuée par une délégation japonaise en RDA, on demande à celui qui la conduit ce qui a le plus impressionné les membres de la délégation.

«Ce qui nous a plus impressionnés, dit le chef de la délégation, ce sont vos merveilleux musées : Pergamon, Rodotron, Pentacon...»)

Il n'y a aucune difficulté linguistique à traduire cette histoire. Et pourtant elle est incompréhensible à qui ne sait pas que Pergamon est le nom d'un important musée d'antiquités à Berlin (comparable au Louvre), que Robotron est le nom d'une usine d'ordinateurs et que Pentakon est une firme de matériel optique. La similarité des terminaisons des noms, et l'aspect vieillot, antique, des usines a favorisé le rapprochement dans les esprits japonais.

16. Ein Mann ist in den Westen übergesiedelt. Er geht in einen Laden und sagt : «Guten Tag, ich möchte eine Schachtel Cabinet.» «Hammwanich», sagt die Verkäuferin.

«Geht das schon wieder los!?», stöhnt der Mann.

(Traduction proposée :

Un homme vient de passer à l'Ouest. Il entre dans un débit de tabac et dit: «Bonjour, je voudrais un paquet de Cabinet, s'il vous plaît.

- Y-en-a-pas! dit la vendeuse.

- Oh non, ça ne va pas recommencer !» dit l'homme excédé.)

La réponse du marchand est banale à l'Ouest et aussi banale à l'Est. Mais pour des raisons différentes. On se dit qu'à l'Ouest un manque est une question de hasard tandis qu'à l'Est le manque est une habitude quotidienne, et la réponse du vendeur un stéréotype. C'est précisément à cela qu'a voulu échapper cet homme en passant à l'Ouest.

Au-delà de cette constatation, il convient de rappeler qu'à l'Est on vend des cigarettes de l'Ouest tandis qu'à l'Ouest on ne vend pas les cigarettes de l'Est. (Les Cabinet sont des cigarettes de l'Est). Le choix est donc, de fait, plus grand à l'Est. Ce qui est contraire aux préjugés existant sur l'Est et l'Ouest.

17. In der Sowjetunion wird ein neues Flugzeug erprobt. Doch jedesmal, bevor der Prototyp sich überhaupt vom Boden lösen kann, brechen die Flügel genau am Rumpf ab. Jedesmal wird der ganze Apparat neu berechnet, jedesmal sind die Berechnungen fehlerfrei, jedesmal brechen die Flügel am Rumpf ab. Da wird in der DDR angefragt, ob man nicht eine Lösung für das Problem finden könne. 
Ein Ingenieur aus Dresden kommt nach zwei Wochen angereist, geht in der Hangar, wo der Prototyp steht, und schließt sich ein. Nach einer Stunde kommt er wieder heraus and läßt die Maschine aufs Rollfeld bringen.

Das Flugzeug startet, hebt ab, ohne daß die Flügel brechen. Das Flugzeug fliegt, und es landet, die Flügel bleiben dran.

«Prachtkerl!», rufen die sowjetischen Techniker und fragen: «Wie haben Sie das bloß gemacht!?»

"Ganz einfach», sagt der Ingenieur aus Dresden, «ich habe die Tragflächen parallel zurn Rumpf perforiert - Loch neben Loch: System Klopapier, reißt garantiert nie an der Stelle, wo's reißen soll!»

(Traduction proposée :

La scène se passe en Union Soviétique où ont lieu les essais d'un nouvel avion. Mais chaque fois, avant même que l'appareil ne décolle, les ailes se détachent à la droite du fuselage. Chaque fois les calculs sont refaits complètement, chaque fois ceux-ci sont exempts d'erreurs et chaque fois les ailes se brisent le long du fuselage. Finalement on décide de consulter la RDA pour trouver une solution au problème.

Un ingénieur de Dresde arrive au bout de quinze jours, va au hangar où se trouve le prototype et s'y enferme. Il en ressort une heure plus tard et fait sortir l'appareil sur la piste. L'appareil roule sur la piste, décolle. Et les ailes ne s'arrachent pas. Il vole et atterrit, les ailes toujours en place.

«Chapeau!» s'écrient les techniciens soviétiques.

«Comment avez-vous fait?» demandent-ils.

«C'est simple comme bonjour, dit l'ingénieur est-allemand. J'ai perforé les ailes parallèlement au fuselage, trou contre trou. Système papier toilette. Ça ne se déchire jamais là où c'est prévu. Garanti.»

Si l'on ne connaît rien sur les pays de l'Est, on comprend que les Allemands sont plus inventifs que les Soviétiques. On comprend également qu'une technique simple est quelquefois plus efficace qu'une technologie complexe. Si l'on sait quelque chose sur ces pays, on se souvient que pendant de longues années, et ce jusque vers les années soixante, la Russie a interdit à ses pays satellites d'avoir aucune industrie de pointe sur leur territoire, aucun avion ne pouvait être produit ailleurs que sur le territoire soviétique (malgré toutes les capacités et les traditions industrielles de certains de ces pays). On se souvient aussi que le papier de toilette a longtemps été un produit rare en Allemagne démocratique et, qui plus est, un produit de très mauvaise qualité lorsqu'il y en avait. C'est en faisant la somme de ce genre de connaissances que l'on possède la toile de fond nécessaire à une bonne compréhension. Pour qu'une traduction soit «fidèle», toute cette toile de fond doit être connue du locuteur de la langue-cible. Traduire la lettre ne fournit pas forcément ces éléments d'information 12 .

Toutes ces blagues politiques supposent des connaissances préalables. Pour les rendre compréhensibles à un auditeur d'une autre langue il faut s'assurer qu'il possède les connaissances nécessaires. C'est bien parce qu'on n'a pas toujours ces connaissances qu'on a en général tant de mal à comprendre les émissions radio ou les journaux satiriques étrangers par exemple. De façon semblable, les blagues éthniques nationales supposent connue une somme de préjugés nationaux (qu'on y adhère ou non, l'essentiel étant de savoir comment situer le conteur et ses personnages). C'est ainsi que les «histoires belges» françaises seront traduites par des histoires polonaises aux États-Unis et pourront apparaître comme des histoires irlandaises en Grande-Bretagne.

L'enseignement des langues étrangères aurait beaucoup à faire en utilisant les histoire drôles et en rendant la compréhension de l'humour de langue seconde possible. 
Nous proposons ici un classement des types de connaissances communes au locuteur et à l'auditeur nécessaires pour la compréhension des blagues, plaisanteries, histoires drôles racontées :

$1^{\circ}$ Références précises des mots (et en particulier pour les langues où les références extra-linguistiques de l'une sont inexistantes pour l'autre).

$2^{\circ}$ Connotations précises des mots (et en particulier dans les cas où les connotations liées à une référence pour une langue n'ont rien de commun avec celles liées à la référence correspondante de l'autre langue).

$3^{\circ}$ Homonymies, ambiguittés, doubles sémantismes de chaque langue.

$4^{\circ}$ Perception de ressemblances phoniques.

$5^{\circ}$ Mentalités, comportements, traits psychologiques propres ou donnés pour propres à un groupe linguistique.

$6^{\circ}$ Types de textes, types de styles, types de publications propres à un groupe linguistique.

$7^{\circ}$ Valeurs (morales, religieuses, scientifiques, etc.) qui imprègnent les locuteurs d'une langue.

$8^{\circ}$ Environnement social, politique, économique, d'un groupe linguistique (actualité et histoire).

Cette liste n'est pas exhaustive ; elle résume les présupposés cognitifs des situations de traduction de l'humour que nous avons rencontrées à ce jour.

L'enseignement des langues vivantes étrangères pourrait s'appuyer systématiquement sur la compréhension et la traduction de l'humour. On pourrait concevoir une progression du début de l'initiation à une langue seconde jusqu'au perfectionnement et à la pratique (quelle masse de connaissances et de pratique faut-il avoir pour créer spontanément des calembours en langue seconde?). A un niveau élémentaire, des tentatives ont été faites pour utiliser les blagues en classe de langue. «Une blague par jour» était l'objectif. Le résultat atteint fut une acquisition plus rapide de vocabulaire et une sensibilisation évidente à des façons d'appréhender les choses qui varient de peuples à peuples $^{13}$. À un niveau intermédiaire, certains cours de version ou thème utilisent les textes humoristiques d'auteurs célèbres. Ceci n'est ni fréquent ni systématique. Par une progression des difficultés fondée sur les huit catégories définies plus haut, on pourrait amener les élèves à plus de réceptivité à l'égard de l'humour de l'autre. (Et on les amènerait en même temps à prendre plus de distance à l'égard d'eux-mêmes, ainsi qu'à apprendre en s'amusant.) Nous n'avons présenté ici que des histoires drôles courtes; ce n'est que l'une des pistes possibles. Les billets d'humeur - plus longs - en sont une autre. Les enregistrements de «chansonniers» (ou leurs équivalents, satiristes, émissions satiriques radiophoniques), en seraient aussi une, pour étudiants plus avancés, car l'oral est toujours plus difficile à travailler que l'écrit. Les pièces de théâtre (par exemple de Jules Romains ou de Marcel Pagnol) constitueraient une autre étape. Les romans (bien rares actuellement) fourniraient une ample matière pour étudiants spécialisés. La presse, peut-être ce qu'il y a de plus difficile à comprendre parce que reposant sur une actualité en perpétuel changement, pourrait fournir des textes de haut niveau de difficulté pour des étudiants traducteurs (par exemple le Canard enchaîné). Les dessins peuvent être utilisés tout aussi bien que les textes au sens propre. L'humour d'Astérix peut être analysé à différents niveaux et des traductions peuvent être trouvées qui rendent compte seulement des jeux de langue ou qui rendent compte aussi des allusions historiques, sociales, culturelles propres à chaque registre de la vie française dépeinte dans chacun des volumes. De la même façon les bandes dessinées argentines de Quino dépeignant la vie et les réflexions de Mafalda sont des textes pouvant être très simples ou très complexes selon la somme de connaissances culturelles que l'on veut faire passer dans la traduction ${ }^{14}$. 
Notes

* Cet article a, dans sa première version, donné lieu à une communication au Congrès de la Modern Language Association (MLA Convention) à New York, le 30 décembre 1986 dans le cadre de la session "Humor and Language Theories III : Applications in Literature» présidée par Don L.F. Nilsen sous le titre : «Humor and Translation». Plusieurs exposés ont ensuite été donnés sur ce thème (avec accent sur les questions de traduction ou les questions pédagogiques selon le cas, à New Mexico State University, Las Cruces, NM (rencontre avec des enseignants et des traducteurs organisée par Jacques Laroche, professeur au département de français de cette université), à Arizona State University, Tempe, AZ (exposé devant des enseignants de divers départements de langues vivantes à la demande de William Hendrickson et Owen Wollam, professeurs au département de français, et avec l'aide de Don L.F. Nilsen, professeur au département d'anglais et organisateur des congrès WHIM), dans le cadre des soirées-rencontres de l'Alliance française de Phoenix-Scottsdale, Arizona, organisées par Francine Verhaagen, devant un public d'enseignants et de professionnels de divers domaines. Ces trois exposés ont eu lieu en avril et mai 1987.

* Vendre cet éléphant blanc au marché noir ne nous est pas apparu comme une solution satisfaisante à cause de la connotation de clandestinité de ce type de marché probablement incompatible avec la présence d'un animal d'une telle dimension.

1. Robert Escarpit (1960) : l'Humour, PUF, Que sais-je ?, 1 re éd.

2. Des MacHale (1982): The Book of Elephant Jokes, Dublin \& Cork, Mercier.

3. Solution proposée par Lucie Laurian.

4. Herb True (1981) : Funny Bone Too!, South Bend, IN, American Humor Guild.

5. Solution proposée par les étudiants du séminaire «Stylistique contrastive» dirigé par A.-M. Laurian à l'Institut de linguistique de l'Université de la Sorbonne Nouvelle - Paris III, 1987.

6. Toute proposition qui nous serait envoyée serait la bienvenue (tant pour cet exemple particulier que pour d'autres cas que nous pourrions soumettre à nos éventuels correspondants).

7. Arthur Bloch (1984): Murphy's Law - and Other Reasons why Things Go wous, Los Angeles, Price/ Stern/Sloan.

8. Voir Anne-Marie Loffler-Laurian (1980): «L'expression du locuteur dans les discours scientifiques. Je nous et on dans quelques textes de physique et de chimie», Revue de linguistique romane, no 173-174.

9. Voir Anne-Marie Loffler-Laurian (1984): «Normes de communication et traduction de textes techniques», $M E T A$, vol. $29, \mathrm{n}^{\circ} 2 ;(1985)$ : «Traduction automatique et style», Babel, n० $2 ;(1986)$ : «Post-édition rapide et post-édition conventionnelle : deux modalités d'une activité spécifique», Multilingua, 5-2 et 5-4.

10. Kenneth Edwards (1977) : I Wish I'd Said that too !, London, Abelard.

11. Clement de Wrobleswsky (1986): Wo wir sind ist worn-der politische Witz in der DDR, Hamburg, Rasch und Röhring.

12. Les relations entre information et style ont été discutées par ailleurs, voir Anne-Marie Loffler-Laurian (1986): «Recherches lexicales et syntaxiques sur les discours scientifiques et techniques», Thèse pour le doctorat d'État, Université de la Sorbonne Nouvelle - Paris III.

13. Processus expérimenté dans le cadre d'un établissement d'enseignement secondaire parisien.

14. Voir Isabel Santi (1983) : «Quelques remarques sur la difficulté de traduire l'effet comique - Étude d'un cas : la bande dessinée 'Mafalda'», Contrastes, n 6 . 